\title{
Ein sparsames länderproporzoptimierendes parteienproporz- gewährendes automatisches Mandatszuteilungsverfahren mit Ausgleich ohne negatives Stimmgewicht
}

\author{
Joachim Behnke
}

Am Mittwoch, dem 25. Juli 2012, hat das Bundesverfassungsgericht das erst zehn Monate zuvor vom Bundestag verabschiedete neue Wahlgesetz für verfassungswidrig erklärt und zwar in mehreren Punkten. Mit diesem Gesetz hatte die Koalition auf das Urteil des Bundesverfassungsgerichts vom 3. Juli 2008 reagiert, in dem dieses das vorherige Wahlgesetz wegen des Effekts des so genannten negativen Stimmgewichts für verfassungswidrig erklärt hatte. Dabei handelt es sich um das Phänomen, dass mehr Stimmen für eine Partei sich in weniger Mandaten für sie auswirken können oder umgekehrt.

Das neue Wahlgesetz, das nach mehr als drei Jahren Beratung fertiggestellt und von der Regierungsmehrheit gegen den Willen der Opposition verabschiedet worden war, wurde vom Verfassungsgericht als „ernüchternd“ ${ }^{1}$ bezeichnet, was man wohl getrost als kaum verhohlene, massive Kritik an den auch handwerklich massiven Mängeln des neuen Gesetzes deuten mag. Dieses wurde in drei Punkten für verfassungswidrig erklärt. ${ }^{2}$

(1) Das Auftreten von negativen Stimmgewichten wurde dadurch zu verhindern gesucht, dass die Listenverbindungen der Parteien aufgebrochen werden. Zuerst werden die insgesamt zu vergebenden Sitze auf die Länder verteilt, dann innerhalb der Länder auf die Landeslisten der verschiedenen Parteien. Die grundsätzliche Vorgehensweise wurde vom BVerfG als zulässig betrachtet, nicht aber die Berechnung der Landeskontingente anhand der Wählerzahlen. Da diese unabhängig von den Wahlentscheidungen der Bürger nicht feststehen, kommt es so zu einer neuen Form des negativen Stimmgewichts.

(2) In einer zweiten Zuteilungsrunde wurden im neuen Wahlgesetz „Reststimmen“ gesammelt und auf dieser Berechnungsbasis so genannte Zusatzmandate vergeben. Die Reststimmen bezogen sich dabei auf die positiven Überschüsse der Stimmenzahlen einer Partei zu der innerhalb eines Bundeslands benötigten Stimmenzahl für die Anzahl der Sitze, die sie dort erhalten hat. Da so bei der Reststimmenverwertung nur Abrundungseffekte, die zuvor im Rahmen der Sitzzuteilung nach Sainte-Laguë aufgetreten sind, berücksichtigt werden, aber nicht die Aufrundungseffekte, die ja ebenfalls stattfinden, führt das Verfahren lediglich zu einer Vergabe zusätzlicher Sitze, ohne seinen eigentlichen Zweck, den Ausgleich von unnötiger Disproportionalität, zu verwirklichen. Die Reststimmenverwertung ist daher überflüssig und in der im neuen Wahlgesetz verwandten Art sogar unzulässig, da sie zu einer Doppelgewichtung bestimmter Stimmanteile führt. Eine Reststimmenverwertung, die Aufund Abrundungseffekte berücksichtigt, wäre zulässig, ist aber nicht geboten.

(3) Im neuen Gesetz wurde in keiner Weise versucht, das Problem der Überhangmandate zu lösen, die den „Grundcharakter der Verhältniswahl“ aufzuheben vermögen, wenn sie in hinreichend großer Zahl auftreten. Das Verfassungsgericht hat erneut und mit noch stärkerer Akzentuierung als in vorhergehenden Urteilen deutlich gemacht, dass die Über-

1 So der Präsident Andreas Voßkuble bei der Verkündung der Entscheidung am 25. Juli 2012.

2 Vgl. BVerfG, 2 BvF 3/11 vom 25. Juli 2012; siehe auch den Aufsatz von Volker M. Haug in diesem Heft der ZParl. 
hangmandate grundsätzlich ein Problem sind, da sie die Erfolgswertgleichheit der Wählerstimmen und damit die Chancengleichheit der Parteien verletzen. In seinem Urteil hat das BVerfG allerdings eine „Schmerzgrenze“ definiert, bis zu der die Überhangmandate hinzunehmen seien. Diese wurde auf 15 Mandate festgelegt. Dieser Wert ergibt sich daraus, dass er der Hälfte der Anzahl von Mandaten entspricht, die zur Bildung einer Fraktion benötigt werden.

Das Verfassungsgericht hat trotz der knapp bemessenen Zeit bis zur nächsten Bundestagswahl darauf verzichtet, selbst eine Art von Übergangsregelung für die nächste Bundestagswahl festzulegen, sondern diese Aufgabe an den Bundestag zurückgegeben. Es hat auf seine eigene Rolle verwiesen, lediglich die äußeren Beschränkungen zu setzen, innerhalb derer der Gesetzgeber tätig werden kann. Der Ball liegt also im Feld des Parlaments, das nun ein Gesetz vorzulegen hat, das sowohl dem Urteil von 2008 über das negative Stimmgewicht als auch der neuen Judikatur Rechnung trägt.

Wahlsystemfragen sind immer auch Machtfragen. Da es hierbei um die originäre Verteilung der Regierungsgewalt selbst geht, sind die machtpolitischen Interessen der Parteien bei Wahlgesetzen sogar unmittelbarer berührt als bei jedem anderen Gesetz. Es wäre daher naiv zu vermuten, dass die Parteien ihre Interessen ausgerechnet bei Wahlsystemfragen ganz und gar außer Acht lassen. Der Realismus legt nahe, hier keine idealistisch geprägte Politik zu erwarten, sondern "politics without romance“"3, innerhalb derer reale Akteure mit realen Präferenzen ebendiese im bestmöglichen Sinn durchzusetzen versuchen. Dennoch gibt es ein gutes Argument, warum die Parteien hier tatsächlich von einer Verfolgung ihrer engen Interessen absehen sollten: Machtinteressen sollten in einer Demokratie genau dann nicht ins Spiel gelangen, wenn es um die Regeln der Machtverteilung selbst geht. Das Wahlgesetz aber ist ein elementarer Bestandteil dieser grundlegenden Spielregeln der Demokratie und wird aus eben diesem Grund ja auch häufig als materielles Verfassungsrecht bezeichnet.

\section{Die Minimallösung einer auf das Notwendige beschränkten Neufassung des Gesetzes}

Die Parteien können nun wieder wie nach dem Urteil von 2008 grundsätzlich auf zweierlei Weise reagieren, das heißt zweierlei Haltungen zum Reformprozess an den Tag legen. Die eine, hier der Einfach halber die positivistische genannt, besteht darin, ein neues Gesetz zu entwerfen, das lediglich darauf gerichtet ist, verfassungsfest zu sein. Man würde dafür auf das vorliegende neue oder das alte Gesetz als Referenz zurückgreifen und von dort aus nur jene Modifikationen vornehmen, die als notwendig erachtet werden, um die Verfassungskonformität zu gewährleisten. Fragen der Eleganz, der Verständlichkeit und der internen Kohärenz sind nach dieser Sichtweise sekundär und können vernachlässigt werden - selbst die Frage, inwieweit ein solches Gesetz demokratietheoretischen Erfordernissen und allgemeinen Fairnessmaßstäben gerecht würde -, solange diese durch die Verfassung nicht zwingend geboten sind. Die zweite Haltung würde nicht die Urteile des Verfassungsgerichts selbst als den in erster Linie zu verwirklichenden Maßstab ansehen, sondern diese als zu beachtende Nebenbedingungen beim Design eines gelungenen Wahlsystems betrachten. Dafür gibt

3 James M. Buchanan, Politics without Romance: A Sketch of Positive Public Choice Theory and its Normative Implications, in: ders. / Robert D. Tollison (Hrsg.), The Theory of Public Choice, Ann Arbor 1984, S. $11-22$. 
es inhaltliche Kriterien, die nicht direkt aus der Verfassung abgeleitet werden können und daher von dieser unabhängig sind. Diese Haltung soll daher als design-orientiert bezeichnet werden. Die beiden Haltungen unterscheiden sich hinsichtlich ihrer Prioritätensetzung und führen in gewissen Aspekten zu unterschiedlichen Bewertungen. Ein schlechtes Gesetz im Sinne des Designs ist nicht zwangsläufig verfassungswidrig, und ein verfassungskonformes Gesetz beinhaltet nicht notwendigerweise ein ansprechendes Design.

Man kann also gewissermaßen zwischen den Bedingungen unterscheiden, die ein Wahlgesetz erfüllen muss, und denen, die ein vom Design her gelungenes Wahlgesetz erfüllen sollte. Abgesehen von den selbstverständlichen, verfassungsgemäßen Grundsätzen, die in jedem Fall einzuhalten sind, wären die Muss-Bedingungen die folgenden:

(1) Das Wahlsystem muss den Effekt des negativen Stimmgewichts beseitigen oder zumindest auf sehr seltene Fälle beschränken, die als unvermeidbare Folge anderer technischer Eigenheiten des Systems (zum Beispiel Rundungseffekte) auftreten.

(2) Die Anzahl der Überhangmandate darf maximal 15 betragen. (Diese Muss-Bedingung ist eine konditionale, da sie angesichts der Festlegung des Wahlsystems auf den Typus der personalisierten Verhältniswahl gilt. Der Gesetzgeber wäre hier aber grundsätzlich frei, sich für einen anderen Typus zu entscheiden, ist aber eben nicht frei, sich von den logischen Folgen seiner einmal getroffenen Entscheidung für einen bestimmten Typus von Wahlsystem zu lösen.)

Anhänger der positivistischen Haltung könnten also das eben gescheiterte Gesetz zur Vorlage nehmen und es entsprechend der Kritik des BVerfG verfassungskonform machen. Hierfür müssten die folgenden Änderungen vorgenommen werden:

(1) Die Landeskontingente werden anhand der Wahlberechtigten (alternativ anhand der Bevölkerungszahlen) berechnet. ${ }^{4}$

(2) Die Reststimmenverwertung entfällt. (Alternativ könnte man auch eine Reststimmenverwertung einführen, die Auf- und Abrundungseffekte berücksichtigt. Da diese am Verteilungsergebnis bei gegebener Stimmenzahl nur geringfügige Änderungen herbeiführen würde, kann sie für die folgenden Berechnungen vernachlässigt werden.)

(3) Ein Ausgleich der Überhangmandate findet erst ab dem 16. Überhangmandat statt. Das heißt die Ausgleichsregelung findet erst dann Anwendung, wenn mehr als 15 Überhangmandate anfallen, und sie bezieht sich dann auch nur auf die Differenz der Anzahl der Überhangmandate zur zulässigen Höchstzahl. Die ersten 15 Überhangmandate verbleiben gewissermaßen als „Bonus“, der über den Ausgleich hinweg beibehalten wird. (Ob man ein solches Verfahren noch berechtigt als „Ausgleich“ bezeichnen könnte, sei dahingestellt.)

4 Tatsächlich war dies eine der handwerklich gröbsten Fahrlässigkeiten im neuen Gesetz, die voraussehbar zu verfassungsrechtlichen Problemen hinsichtlich des negativen Stimmgewichts führen musste. Durch die Orientierung der Berechnung der Sitzkontingente der Länder an der Wahlbeteiligung kann es wieder aufgrund der Wahlentscheidungen der Bürger zu einer Verschiebung von Mandaten zwischen verschiedenen Landeslisten kommen und damit auch zu einem negativen Stimmgewicht. Dies hätte sich leicht verhindern lassen, indem man vor der Wahlentscheidung unabhängige Größen wie die Bevölkerungszahlen oder die der Wahlberechtigten genommen hätte, um die Sitzkontingente festzulegen, vgl. Joachim Behnke, „Dies ist das Wahlsystem meines Missvergnügens “ - Eine Kritik des Entwurfs der CDU/CSU und FDP für eine Reform des Wahlgesetzes, 26. August 2011, http://www.zu.de/deutsch/lehrstuehle/politikwissenschaft/CDU_ Wahlreformentwurf.pdf (Abruf am 20. August 2012). 
Der kritischste Punkt an einer solchen minimalen Modifikation des Gesetzes der Regierungskoalition wäre ohne Zweifel der Ausgleich erst ab dem 16. Überhangmandat, der im Folgenden als verkürzter Ausgleich bezeichnet wird. Zielt man von vornherein nur auf einen solchen, dann kämen als Ausgangspunkte für das neue Gesetz die Entwürfe von Grünen, SPD und Linke nicht in Frage, weil diese bis auf die darin vorgesehene Behandlung der Überhangmandate mit dem alten Gesetz identisch wären, solange weniger als 16 Überhangmandate anfielen, womit das Problem des negativen Stimmgewichts weiterhin bestehen bleiben würde.

Ein verkürzter Ausgleich wäre in mehrerlei Hinsicht äußerst problematisch, und zwar sowohl in normativer Hinsicht als auch unter dem Aspekt der technischen Umsetzung. Überhangmandate werden im Urteil - durchaus in Kontinuität zur vorhergehenden Rechtsprechung $^{5}$ - als ein bis zu einem gewissen Umfang hinzunehmendes Übel charakterisiert. Nirgends argumentiert das Gericht aber, dass die Überhangmandate als solche in irgendeiner Weise einen legitimen Anspruch auf Sitze darstellen könnten. Dieser entsteht lediglich dadurch, dass sie als Direktmandate gewonnen werden. Die Erfüllung dieses Anspruchs muss aber keineswegs zwangsläufig zu Überhangmandaten führen. Es ist daher logisch daraus nicht abzuleiten, dass die Überhangmandate einen weitergehenden Anspruch auf Mandate als den aufgrund der Zweitstimmen bestehenden begründen könnten. ${ }^{6}$ Ein solcher, vom Verhältnisausgleich gewissermaßen losgelöster und eigenständiger Anspruch wurde aber letztlich von den Vertretern der Regierungskoalition behauptet. In dieser Interpretation steht der Erststimme gewissermaßen ein unabhängiger, legitimer Einfluss auf die Zusammensetzung des Parlaments zu, so dass dementsprechend auch kein Ausgleich nötig ist. Das Gericht hat sich dieser Ansicht nicht angeschlossen, sondern sieht den Ausgleich ab einer gewissen Anzahl von Überhangmandaten sogar als zwingend geboten an.

Das BVerfG hat also die Überhangmandate einerseits zwar als grundsätzlich problematisch charakterisiert, aber andererseits leider die Korrektur oder ihren Ausgleich nicht als zwingend erforderlich vorgeschrieben. In dieser Hinsicht weist das Urteil eine gewisse Ambivalenz auf und ist so weder „Fisch noch Fleisch“ geworden. ${ }^{7}$ Doch ein verkürzter Ausgleich, der erst ab dem 16. Überhangmandat durchgeführt würde, wäre demokratietheoretisch höchstproblematisch, denn die größte von Überhangmandaten ausgehende Gefahr einer Mehrheitsumkehrung besteht fast unvermindert auch bei einer Obergrenze von 15 . Drei der sechs Regierungskoalitionen seit 1990 hatten, berücksichtigt man nur die Proporzmandate, einen Vorsprung von weniger als 15 Mandaten vor der Opposition. Grob geschätzt kann man also davon ausgehen, dass die Wahrscheinlichkeit, dass durch zehn bis fünfzehn Überhangmandate neue Regierungsmehrheiten entstehen oder bestehende zerstört werden, ungefähr in der Größenordnung liegt, mit der man eine Sechs beim Würfeln erhält. Wie

5 Vgl. Hans Meyer, Die Zukunft des Bundestagswahlrechts, Baden-Baden 2010.

6 Zur Legitimationsproblematik von Überhangmandaten vgl. Joachim Behnke, Grundsätzliches zur Wahlreformdebatte, in: APuZ, B 4 / 2011, S. 14 - 21, S. 18 ff.

7 Es spricht Einiges dafür, diese Ambivalenz vor allem auf den Wunsch des Gerichts zurückzuführen, zu einem einstimmigen Urteil zu gelangen. Geht man davon aus, dass vermutlich allgemeiner Konsens darüber bestand, dass Überhangmandate in Fraktionsstärke beziehungsweise in der Größenordnung von fünf Prozent der Mandate auf keinen Fall hinzunehmen seien, und ein gewisser Anteil der Richter sogar einen Ausgleich der Überhangmandate vom ersten an als erforderlich ansah, dann sind die 2,5 Prozent der Mandate, denen die „magische“ 15 ja entspricht, der klassische Kompromiss, dem eine Verhandlungslösung in der Mitte entspricht. 
aber jeder vom „Mensch ärgere dich nicht“ weiß: Früher oder später kommt die Sechs immer. Zwar ist es angesichts aktueller Umfragewerte eher unwahrscheinlich, dass Überhangmandate einer schwarz-gelben Koalition erneut zur Mehrheit verhelfen könnten; deutlich realistischer aber ist ein Szenario, bei dem für die CDU anfallende Überhangmandate eine ansonsten bestehende Mehrheit von Rot-Grün wieder zunichte machen oder eine eigentlich nicht bestehende Mehrheit von Rot-Grün entstehen lassen könnten, wenn die Überhangmandate vor allem im rot-grünen Lager anfallen. Die Obergrenze von 15 zulässigen, nicht auszugleichenden Überhangmandaten ist daher als eine äußerst unglückliche Lösung zu betrachten. Die Setzung der konkreten Zahl 15 wirkt willkürlich, und die Begründung ist nicht stichhaltig, denn der „Grundcharakter der Verhältniswahl“ wird ja nicht desto stärker verletzt, je mehr Überhangmandate anfallen, sondern je mehr die Sitzzahlenverhältnisse zwischen den Parteien von den Stimmenverhältnissen abweichen. 13 Überhangmandate für eine Partei allein, wie zum Beispiel 1998 für die SPD, verzerren die Proportionalität stärker als zehn Überhangmandate für die CDU und acht für die SPD. Hält man sich strikt an die Obergrenze, hätte das zur Konsequenz, dass im Effekt weniger dramatische Verzerrungen durch die Überhangmandate ausgeglichen würden, während wesentlich stärkere Verletzungen des „Grundcharakters der Verhältniswahl“ folgenlos blieben.

Es gibt zudem einen klaren Hinweis, dass solche Veränderungen der Mehrheit vom Gesetzgeber nicht erwünscht sind beziehungsweise der mit dem Design verfolgten Intention widersprechen. Das alte und das neue Wahlgesetz sowie alle sonstigen Entwürfe enthalten die so genannte Mehrheitsklausel, nach der eine Partei, die eine Mehrheit der zu berücksichtigenden Zweitstimmen erhält, in jedem Fall auch eine Mehrheit der Sitze erhalten muss. Das Prinzip der Mehrheitstreue, wie es in der Mehrheitsklausel verankert ist, stellt - auch wenn der intuitive erste Eindruck ein anderer sein mag - ein klares Bekenntnis zum Proporzcharakter des Wahlsystems dar. Es erzwingt zwar die Erhaltung des Proporzes nur für einen speziellen Fall, verrät damit aber eine normative Grundhaltung, die über diesen Fall hinaus zu interpretieren ist. ${ }^{8}$ Dieses Prinzip der Mehrheitstreue oder Mehrheitserhaltung bedeutet daher - konsequent auf ein Verhältniswahlsystem übertragen -, dass auch jede Koalition von Parteien, die eine Mehrheit der zu berücksichtigenden Zweitstimmen erhalten hat, eine Mehrheit an Sitzen erhalten sollte. Dies erscheint auch aus Gründen der Legitimation erforderlich.

Die Legitimität einer Regierung beruht nicht lediglich darauf, dass sie die Sitzmehrheit hinter sich hat. Nicht die Verfahren an sich schaffen die Legitimation, sondern die allgemeine Akzeptanzfähigkeit der Verfahren. Wer diese allgemeine Akzeptanzfähigkeit verletzt, unterhöhlt damit auch die Kraft des Verfahrens, Legitimität herzustellen. ${ }^{9}$ Es ist schwer vorzu-

8 Reine Mehrheitssysteme wie das englische oder das us-amerikanische verfügen nicht über eine solche Einschränkung. Ergebnisse, bei denen nach Stimmen die zweitgrößte Partei eine Mehrheit der Sitze erhält, werden zwar bezüglich des englischen Systems in der Literatur als „perverse outcomes“ (Paul Mitchell, The United Kingdom. Plurality Rule under Siege, in: Michael Gallagher I ders. (Hrsg.), The Politics of Electoral Systems, Oxford 2005, S. 157 - 184, S. 173) bezeichnet, es gibt aber keine institutionellen Vorkehrungen dagegen und sie werden als vom Design zwar ungewünschte, aber hinzunehmende Effekte betrachtet. Auch die amerikanische Bevölkerung scheint es mit einer gewissen Gelassenheit zu nehmen, wenn der Sieger der Wahl, wie zum Beispiel im Jahr 2000 George W. Bush, nicht die „popular vote“ auf sich vereinigen kann.

9 Vgl. Wilfried Hinsch, Legitimacy and Justice. A Conceptual and Functional Clarification, in: Jörg Kühnelt (Hrsg.), Political Legitimization without Morality?, Berlin 2008, S. 39 - 52. 
stellen, dass eine Regierung, die sich auf weniger Wählerstimmen berufen könnte als die Opposition, von den deutschen Wählern als legitim empfunden würde. Wäre 2011 bei der Wahl in Baden-Württemberg ein analoges Verfahren zum verkürzten Ausgleich angewandt worden, wäre die schwarz-gelbe Regierung unter Stefan Mappus im Amt geblieben, obwohl die Oppositionsparteien mehr Stimmen erhielten. Ein solches Ergebnis wäre dem Glauben der Bürger, dass ihr Wahlsystem demokratieadäquat sei, sicherlich nicht förderlich gewesen.

Ein Verfahren, das gegen den „Geist der Institution“10 verstößt, unterminiert nicht nur das durch das Verfahren hervorgebrachte Ergebnis, sondern letztlich auch das Ansehen des Verfahrens selbst. Die Erfüllung des Prinzips der Mehrheitstreue ist daher eine weitere normative Forderung, die sich daraus ergibt, dass das Ergebnis einer Wahl auch von den Wählern, die dieses Ergebnis nicht wollten, akzeptiert werden muss. Die Verpflichtung der unterlegenen Seite auf die Akzeptanz des Ergebnisses aber setzt voraus, dass sie keinen Anlass haben darf, sich dieser Verpflichtung mit berechtigten Gründen zu entziehen. Eine Verletzung des Prinzips der Mehrheitstreue aber würde ihr zweifelsohne einen solchen Grund liefern. Dieses lässt sich in einem Vielparteiensystem nur dann optimal umsetzen, wenn die Verteilung der Sitze zwischen den Parteien in bestmöglicher Weise proportional zur Verteilung der Stimmen ist, denn nur dann ist gewährleistet, dass jede Mehrheit an Stimmen auch eine Mehrheit an Sitzen erhält. Eine strenge Erfüllung des Maßstabs der Erfolgswertgleichheit garantiert daher auch die Einhaltung des Prinzips der Mehrheitstreue.

Dass Überhangmandate in der Lage sind, die Legitimität eines Wahlergebnisses zu gefährden, wird auch durch die Ergebnisse von Bevölkerungsumfragen gestützt. In einem (nicht repräsentativen) Online-Panel der deutschen Wahlstudie GLES zur Bundestagswahl 2009 gab jeweils eine deutliche Mehrheit der Anhänger von SPD, Grünen und Linken an, dass Überhangmandate den Wählerwillen verzerrten. Wenig überraschend sah das im Lager von CDU und FDP nur eine Minderheit so. ${ }^{11}$ Offensichtlich ist die Wahrnehmung also durchaus abhängig davon, ob man davon ausgeht, dass die Überhangmandate für das eigene Lager von Vorteil oder von Nachteil sind. Entscheidend aber ist, dass die Überhangmandate aus Sicht derer, die einen Nachteil davon haben, die Legitimation einer Mehrheit, die sich auf diese Mandate stützen würde, unterhöhlen würden. In derselben Umfrage sprach sich insgesamt nur circa ein Viertel der Befragten für eine Beibehaltung der Überhangmandate bei einer zukünftigen Reform des Wahlgesetzes aus. Selbst im Lager von CDU und FDP gab es keine Mehrheit, die die Überhangmandate erhalten wollte. ${ }^{12}$ Es spricht also Einiges dafür, dass Überhangmandate auch von einem überwiegenden Teil der Bevölkerung als problematisch angesehen werden. ${ }^{13}$

Da wie erwähnt in drei der sechs letzten Bundestagwahlen Überhangmandate in einer Größenordnung von 15 Mandaten bei einem verkürzten Ausgleich genügend Potenzial ge-

10 Reinhard Zintl, Kollektive Entscheidungsprozesse und die Funktionalität legitimer Macht, in: Martin Held / Gisela Kubon-Gilke / Richard Sturm (Hrsg.), Normative und institutionelle Grundfragen der Ökonomik, Marburg 2008, S. 121 - 141, S. 122.

11 Vgl. Jan Eric Blumenstiel / Ossip Fürnberg, Wissen und Einstellungen zu Überhangmandaten, in: ZParl, 43. Jg. (2012), H. 1, S. $132-141$.

12 Vgl. ebenda, S. 139.

13 Gültige Verallgemeinerungen für die Gesamtbevölkerung sind nicht möglich, da es sich nicht um eine repräsentative Zufallsstichprobe handelte. Dennoch stellen auch solche Umfragen durchaus eine starke Evidenz dar, die man nicht unterschätzen sollte. 
habt hätten, um das politische Ergebnis der Wahl zu verändern, wenn nicht sogar umzukehren, würde ein Ausgleich, der erst ab dem 16. Mandat stattfindet, die Anreize zu strategischem Verhalten bei den Wählern und Parteien nicht mindern. Mit Hilfe von Umfragedaten lässt sich schätzen, dass zum Beispiel allein circa sieben der zehn Überhangmandate der CDU in Baden-Württemberg durch Stimmensplitting zustande gekommen sind. ${ }^{14}$ Bedenklich daran ist vor allem, dass nicht mehr nur das typische, klassische Splittingverhalten zu beobachten ist, bei dem die Anhänger kleiner Parteien ihre ansonsten verloren gehende Erststimme dem Kandidaten einer großen Partei geben, sondern dass offensichtlich ein nicht unerheblicher Teil der Anhängerschaft der CDU zumindest bei der letzten Wahl in BadenWürttemberg mit der Zweitstimme eine kleine Partei, nämlich die FDP, gewählt hat. Dabei könnte es sich um einen nicht unproblematischen Lerneffekt aus der Nachwahl von Dresden handeln, dergestalt, dass die Anhänger einer großen Partei, wenn sie sich sicher sein können, dass ihre Partei so oder so Überhangmandate erzielt, ihrer Partei bewusst die Zweitstimme entziehen, da nun diese ansonsten eine verschwendete Stimme wäre. Auch die Anreize für Parteien, sich strategisch zu verhalten, sind demnach groß. Die Überhangmandate der CDU in Baden-Württemberg könnten zum Beispiel relativ leicht durch Wahlkreisabsprachen zwischen SPD und Grünen verhindert werden. Damit aber würde das Endergebnis einer Wahl immer stärker vom mehr oder weniger großen strategischen Geschick der Parteien und ihren jeweiligen Anhängerschaften abhängig sein und dementsprechend immer weniger als Ausdruck des originären Willens der Wähler gedeutet werden können. Ein gelungenes Design sollte daher so wenig Anreize zu strategischem Verhalten bieten wie möglich. ${ }^{15}$

Ein verkürzter Ausgleich wirft aber zusätzlich schwerwiegende technische Probleme auf beziehungsweise lässt sich auf kohärente Weise nur durchführen, wenn man ihn auf eine unnötig teure Art anwendet. Gehen wir also davon aus, dass das Problem der Überhangmandate grundsätzlich durch Ausgleichsmandate gelöst werden soll, wie es zum Beispiel auch in sämtlichen Landeswahlgesetzen geregelt ist, insoweit dort Überhangmandate entstehen können. Üblicherweise wird bei solchen Regelungen die Anzahl der insgesamt zu verteilenden Sitze sukzessive so lange erhöht, bis alle Überhangmandate in normale Proporzmandate aufgehen. Bei einem zweistufigen Verteilungsprozess mit Ober- und Unterverteilung führte dies jedoch zu einer beachtlichen Aufblähung des Bundestags. Hätte man zum Beispiel eine solche Ausgleichsregelung bei der Bundestagswahl 2009 angewandt, bei ansonsten demselben Wahlsystem, wie es seinerzeit gültig war, dann hätte der Bundestag auf 801 Sitze vergrößert werden müssen, um alle 21 Überhangmandate der CDU auszugleichen. Allerdings handelte es sich dabei um einen großen beziehungsweise. doppelten Ausgleich, das heißt sowohl der Parteien- als auch der Länderproporz würden dabei gewahrt. Tatsächlich aber ließe sich der Ausgleich wesentlich „billiger" gestalten, wenn man sich auf die Wiederherstellung des durch die Überhangmandate verletzten Parteienproporzes beschränken würde. Dieser „kleine“ Ausgleich würde also nur auf der bundesweiten Ebene der Oberverteilung stattfinden, nicht mehr in der darauf folgenden Unterverteilung, bei der die

14 Vgl. Joachim Behnke / Florian Bader, Strategisches Wählen bei der Bundestagswahl 2009 - gibt es Anzeichen für strategisches Lernen?, in: Bernhard Weßels / Oscar W. Gabriel / Harald Schoen (Hrsg.), Wahlen und Wähler. Analysen aus Anlass der Bundestagswahl 2009, Wiesbaden 2012 (im Erscheinen).

15 In diesem Sinne auch Michael Dummett, Principles of Electoral Reform, Oxford 1997, S. 16. 
Mandate einer Partei den verschiedenen Landeslisten zugeordnet werden. ${ }^{16}$ Inklusive Überhangmandate erhielt die CDU 2009 zum Beispiel 194 Mandate. Es würde also genügen, die Sitzzahl so weit zu erhöhen, um diese 194 Mandate der CDU abzudecken. Dafür müsste die Gesamtsitzzahl nur auf 666 erhöht werden. Die SPD erhielte dann 163 Sitze statt 146, die ihr bei einer Basis von 598 zustanden. Es fielen also 17 Ausgleichsmandate für die SPD an. Diese könnten nun so verteilt werden, dass die 163 Sitze der SPD wieder auf die Landeslisten verteilt werden, so dass sie dann in dieser Verteilung aufgingen. Man könnte aber die Ausgleichsmandate auch so verteilen, dass der durch die Überhangmandate ebenfalls verletzte Länderproporz zumindest wieder etwas verbessert wird. Dazu könnte man die Ausgleichsmandate an diejenigen Länder vergeben, in denen keine Überhangmandate angefallen sind und in denen daher der länderbezogene Erfolgswert aller Wähler in diesem Land unterdurchschnittlich ausfällt. ${ }^{17}$

Beschränkt man sich auf den kleinen Ausgleich zur Gewährung des Parteienproporzes, gehen streng genommen die Überhangmandate nicht in den Proporzmandaten auf, sondern man gleicht lediglich den Vorteil, der durch die Überhangmandate errungen wird, durch entsprechende Ausgleichsmandate aus. Denn wenn man die 194 Mandate, die bei $666 \mathrm{zu}$ vergebenden Sitzen der CDU aufgrund ihrer Zweitstimmen zustehen, entsprechend der Zweitstimmen auf die Landeslisten verteilte, entfielen immer noch zwölf Überhangmandate. Diese werden im kleinen Ausgleich gewissermaßen implizit mit Listenmandaten verrechnet, die bei der Erhöhung von 173 auf 194 Proporzmandate für die CDU erst einmal anfallen würden. So müsste zum Beispiel die Landesliste der CDU in Hessen 17 anstatt 15 Mandate erhalten, wenn die Länder im Rahmen der Unterverteilung 194 statt 173 Mandate erhielten. Aber da es bei den 15 ursprünglichen Listenmandaten bleibt, gehen die zwei zusätzlichen Proporzmandate in der Verrechnung mit den zwölf übrig gebliebenen Überhangmandaten auf. Beim kleinen Ausgleich werden also durch die Erhöhung auf 666 Sitze neun der 21 Überhangmandate dadurch abgebaut, dass sie in neu zugeteilten Proporzmandaten aufgehen; die restlichen zwölf Überhangmandate werden hingegen mit den übrigen zwölf zusätzlichen Proporzmandaten verrechnet, die eben gar nicht erst vergeben werden. Der kleine Ausgleich kann also auf relativ „billige“ Weise vorgenommen werden, weil die implizite Verrechnung von „übrig gebliebenen “ Überhangmandaten mit Listenmandaten in anderen Bundesländern vorgenommen werden kann. Entfällt diese Möglichkeit, zum Beispiel weil bei der CSU keine Landeslisten existieren, mit denen Überhangmandate in Bayern verrechnet werden könnten, dann gibt es die Möglichkeit des kleinen Ausgleichs nicht, und nur der doppelte Ausgleich ist möglich. Sechs bis sieben Überhangmandate der CSU würden den Bundestag dann wohl um mehr als 100 Sitze aufblähen.

Noch einmal deutlich komplizierter aber würde es, wenn man gar nicht alle Überhangmandate abbauen, sondern die Anzahl der nicht ausgeglichenen lediglich auf 15 senken wollte. Im Rahmen des doppelten Ausgleichs müsste die Sitzzahl auf 644 erhöht werden,

16 Vgl. Joachim Behnke, Bundestagswahl 2002. Die Bombe tickt weiter: Das immer noch existierende Problem der Überhangmandate und mögliche Lösungen, in: Jürgen W. Falter / Oscar W. Gabriel/ Bernhard Weßels (Hrsg.), Wahlen und Wähler - Analysen aus Anlass der Bundestagswahl 2002, Wiesbaden 2005, S. S. 459 - 483, S. 479 ff. Dieser Ansatz des kleinen Ausgleichs wird ebenfalls vertreten in Daniel Lübbert / Felix Arndt / Friedrich Pukelsheim, Proporzwahrende Anpassung der Bundestagsgröße - ein Lösungsvorschlag für das Problem der negativen Stimmgewichte bei Bundestagswahlen, in: ZParl, 42. Jg. (2011), H. 2, S. 426 - 435.

17 Vgl. Joachim Behnke, a.a.O. (Fn. 16), S. 480. 
damit nur noch 15 Überhangmandate übrigbleiben, die dann alle für die CDU anfallen; es werden also sechs Überhangmandate der CDU und drei der CSU abgebaut. Ein kleiner Ausgleich hingegen ist technisch schwer umzusetzen, da hierfür bekannt sein müsste, welche der ursprünglichen 24 Überhangmandate auf die 15 herunter geschmolzen werden sollen. Innerhalb des doppelten Ausgleichs ist diese Reihenfolge der Abschmelzung - wie gezeigt - klar determiniert, im kleinen Ausgleich hingegen ist sie unbestimmt. Wollte man einen verkürzten, kleinen Ausgleich vornehmen, müsste man also in einem ersten Schritt die Überhangmandate auf 15 innerhalb eines Verfahrens virtuell abbauen, das die abzuschmelzenden Überhangmandate identifiziert, um dann den eigentlichen Abbau innerhalb eines anderen Verfahrens vorzunehmen. Abgesehen von den normativen Bedenken, muss man sich schon sehr weitgehend von jeglichen ästhetischen Empfindungen befreit haben, um eine solche Vorgehensweise zu befürworten. In diesem Zusammenhang sei auch an die Ermahnung des Bundesverfassungsgerichts in seinem Urteil von 2008 erinnert, das Wahlgesetz auf eine „normenklare und verständliche Grundlage“ zu stellen.

Auch wenn das neue Urteil dies also nicht zwingend vorschreibt, ist unter einer designorientierten Perspektive offensichtlich, dass eine auf das Notwendige beschränkte Modifikation des neuen Gesetzes unzureichend sein muss und nur eine Lösung, die alle Überhangmandate ausgleicht oder in ihrer Wirkung neutralisiert oder sie - am besten - erst gar nicht entstehen lässt, wirklich akzeptabel sein kann.

\section{Vor-und Nachteile verschiedener alternativer Entwürfe}

Ist grundsätzlich ein vollständiger Ausgleich, wenn auch in möglichst „,billiger“ Form, vorgesehen, dann kommen die drei alternativen Entwürfe zur Beseitigung des negativen Stimmgewichts von SPD, Grünen und der Linken alle als potenziell geeignete Designs in Frage. Ihnen allen ist gemeinsam, dass sie das Ausgangsproblem des negativen Stimmgewichts dadurch lösen, dass sie die Überhangmandate ins Visier nehmen, da das negative Stimmgewicht im alten Wahlgesetz über die Entstehung von Überhangmandaten ins Spiel kam. Auf diese Weise können zwei Fliegen mit einer Klappe geschlagen werden, nämlich das des negativen Stimmgewichts und das der Überhangmandate.

Der Entwurf der Grünen ${ }^{18}$, der abgesehen von einer Änderung bezüglich der CSUÜberhangmandate, als Gesetzentwurf schon 2009 eingebracht worden und gescheitert war, verfolgt die Logik so genannter Verrechnungs- oder Kompensationsmodelle. Dabei werden die Überhangmandate der CDU durch Listenmandate in Ländern, in denen keine Überhangmandate anfallen, kompensiert, das heißt diese Listenmandate werden nicht vergeben. Die Verrechnungslogik wird insbesondere seit den 1990ern intensiv diskutiert. ${ }^{19}$ Sie lässt sich besonders elegant anwenden, wenn als Zuteilungsverfahren ein Divisorverfahren wie

18 Vgl. BT-Drs. 17/4694.

19 Stellvertretend vgl. hierzu Helmut Nicolaus, Stimmgewicht und Erfolgschancengleichheit im Wahlverfassungsrecht. Prinzipielle Bemerkungen zur Zählwertgleichheit und zur Inflation der Überhangmandate, in: ZParl, 26. Jg. (1995), H. 2, S. 353 - 371; Christian Naundorf, Der überflüssige Überhang: Reformvorschläge, in: ZParl, 27. Jg. (1996), H. 3, S. 393 - 397; Gerald H. Mann, Die unumgängliche Umkehr bei der Berechnung von Überhangmandaten: Reformvorschläge, in: ZParl, 27. Jg. (1996), H. 3, S. $398-404$. 
d'Hondt oder Sainte-Laguë verwendet wird, da dann die Reihenfolge, in der die Sitze vergeben werden, vom ersten bis zum letzten Sitz feststeht. Man muss dann nur die zuletzt zugeteilten Listenmandate streichen, bis alle Überhangmandate kompensiert sind, oder man vergibt die insgesamt nach Abzug der Direktmandate noch zuzuteilenden Proporzmandate in der angegebenen Reihenfolge auf die Landeslisten sukzessive so lange, bis alle Proporzmandate aufgebraucht sind. Dabei beträgt die „Startausstattung“ jeder Landesliste die Anzahl der Direktmandate, die die Partei in diesem Land erhalten hat. Eine Landesliste erhält eines der noch freien Proporzmandate dabei nur dann zugewiesen, wenn der Gesamtanspruch auf Sitze der dortigen Landesliste die Anzahl der in diesem Land gewonnenen Direktmandate übertrifft. ${ }^{20}$

Kompensationsmodelle haben den großen Vorteil, dass sie in der Regel die Gesamtzahl der Sitze beibehalten. Dies gilt allerdings nur, wenn die Überhangmandate alle interner Natur sind. Externe Überhangmandate, die auf der Ebene der Oberverteilung anfallen, können durch Kompensationsmodelle nicht berücksichtigt werden. Dies trifft grundsätzlich auf die Überhangmandate der CSU zu. Daher sah der Entwurf der Grünen für diese Mandate auch eine Zusatzklausel vor, nach der die überschüssigen, externen Überhangmandate dadurch beseitigt werden, dass die am wenigsten erfolgreichen Wahlkreiskandidaten in entsprechender Anzahl kein Direktmandat erhalten. Dieser Entzug stieß allerdings auf massiven Widerstand der anderen Parteien. In der Tat widerspricht es fundamentalen Fairnessempfindungen, wenn Wahlkreissieger ihren Preis, das Direktmandat, nicht erhalten.

So oder so müssen im Kompensationsmodell die Überhangmandate aber irgendwo abgezogen werden - wenn nicht bei den Direkt-, dann bei den Listenmandaten. Im Fall der Bundestagswahl 2009 hätten die 21 Überhangmandate der CDU durch die einzigen noch über die Liste vergebenen Sitze kompensiert werden müssen, denn die Anzahl von 173 Sitzen, die der CDU aufgrund ihres Stimmenanteils zustand, entsprach auch genau der Anzahl der von ihr gewonnenen Direktmandate. In Nordrhein-Westfalen wären demnach acht Listenmandate, in Niedersachsen fünf nicht vergeben worden. In Brandenburg wären vier von insgesamt fünf Sitzen, die der Liste „eigentlich“ zugestanden hätten, weggefallen, in Bremen das einzige Mandat, das die CDU dort aufgrund ihrer Zweitstimmen überhaupt errungen hatte. ${ }^{21}$ Das Fairnessargument bezüglich der Direktmandate sollte aber auch hier gelten. Es ist schwer vermittelbar, warum Listenmandatsträger in Brandenburg, Niedersachsen oder Bremen zur Kompensation des Überhangs auf ihr Mandat verzichten sollten beziehungsweise zum Verzicht gezwungen werden dürften. Auch wenn der rechtliche Anspruch erst dann bestehen mag, wenn die Sitze den Mandatsträgern in spe auch durch das Gesetz zugewiesen werden: Es besteht unabhängig davon ein moralischer Anspruch auf diese Sitze im Sinne einer Verdiensttheorie. ${ }^{22}$ Ein Wahlkreiskandidat, der im Wettbewerb mit anderen Kandidaten mehr Stimmen als jeder der übrigen Mitbewerber gewinnen konnte, hat sich

20 Vgl. Joachim Behnke, Überhangmandate: Ein (behebbarer) Makel im institutionellen Design des Wahlsystems, in: ZPol, 13. Jg. (2003), H. 3, S. 1235 - 1269, S. 1261 ff.; Joachim Behnke, a.a.O. (Fn. 16), S. 475. Da die Direktmandate als „Startausstattung“ eine zu erfüllende Restriktion beziehungsweise Nebenbedingung darstellen, wird dieses Verfahren auch als „direktmandatsbedingte Divisormethode mit Standardrundung" (vgl. Friedrich Pukelsheim, Bundeswahlgesetz - Nächste Etappe, in: DVBL, 123. Jg. (2008), H. 14, S. 889 - 897) bezeichnet.

21 Vgl. Joachim Behnke, Überhangmandate bei der Bundestagswahl 2009 - das ewige Menetekel, in: PVS, 51. Jg. (2010), H. 3, S. $531-552$.

22 Vgl. David Miller, Principles of Social Justice, Cambridge 1999, S. 131 ff. 
das entsprechende Mandat nach der Logik der angewandten Regeln dieses Wettbewerbs verdient. ${ }^{23}$ Es mag sein, dass der moralische Anspruch im Sinne eines Verdiensts eines Listenkandidaten geringer ist als der eines Direktkandidaten, da er nicht selbst unmittelbar gewählt worden ist. Aber der Landesverband seiner Partei kann seinerseits sehr wohl eine Art von moralischem Anspruch auf das Sitzkontingent erheben, das ihm nach den Zweitstimmen zusteht.

Die Kompensation von Überhangmandaten durch den Wegfall von Direkt- oder Listenmandaten setzt daher nicht nur die üblichen Zuteilungsregeln außer Kraft beziehungsweise revidiert sie, sondern unterhöhlt und missachtet außerdem die Kriterien, nach denen diese Zuteilung ursprünglich erfolgt ist beziehungsweise hätte erfolgen sollen. Es sind aber ebendiese Kriterien, die den Anspruch auf die Sitze erst begründen. Während Überhangmandate eine große Herausforderung für das Gerechtigkeitsempfinden aller Wähler von Parteien darstellen, die keine Überhangmandate gewinnen, verletzt die Kompensation von Überhangmandaten vermutlich das Gerechtigkeitsempfinden der Inhaber der betroffenen Listenplätze, die so nicht ins Parlament einziehen. Die Unstrittigkeit eines Wahlsystems setzt allerdings voraus, dass es die Verletzung von tiefgründenden und berechtigten Gerechtigkeits- und Fairnessvorstellungen ${ }^{24}$ zu vermeiden sucht, wo immer möglich. Auch muss an der politischen Umsetzbarkeit solcher Regelungen, die von den Betroffenen als unfair empfunden werden, gezweifelt werden. Es liegt in der Natur der Sache, dass vor allem sehr starke und mächtige Landesverbände wie die von Nordrhein-Westfalen oder Niedersachsen in einem Kompensationsmodell die Zeche für die Überhangmandate zu zahlen hätten. Eine weitere Soll-Bedingung besteht daher darin, dass ursprüngliche, auf VerdienstKriterien beruhende Mandatsansprüche möglichst respektiert werden. ${ }^{25}$

Ein weiteres Problem von Kompensationsmodellen besteht darin, dass sie mitunter den Länderproporz enorm verzerren können. 2009 wären, wie erwähnt, zum Beispiel vier der fünf Mandate in Brandenburg für die Kompensation von Überhangmandaten verwendet worden. Eine Möglichkeit, diese Verzerrung zumindest zu dämpfen, besteht darin, eine Art Puffer von Listenmandaten zu schaffen, der groß genug ist, dass es durch die Kompensation

23 Dies soll allerdings keineswegs heißen, dass die Regeln der relativen Mehrheitswahl die optimalen Wettbewerbsregeln darstellen. Andere Regelsysteme wie „Alternative Vote“ wären womöglich besser geeignet, den „wahren“ Wahlkreissieger zu finden. Aber die Regeln der relativen Mehrheitswahl sind nun einmal diejenigen, die im Gesetz für den Wettbewerb im Wahlkreis verankert sind.

24 Nicht jede Wahrnehmung von „Ungerechtigkeit“ kann als begründet und berechtigt angesehen werden. Zum Beispiel ist die Ansicht durchaus verbreitet, dass es „ungerecht“ sei, wenn nicht die stärkste Partei automatisch den Auftrag zur Regierungsbildung erhalte beziehungsweise dass eine Regierung ohne die stärkste Partei zustande kommen kann. In einem Verhältniswahlsystem aber hat diejenige Koalition das Recht, eine Regierung zu bilden, die eine Mehrheit an Sitzen auf sich vereinigen kann, unabhängig davon, ob die stärkste Partei daran beteiligt ist oder nicht. Solche Wahrnehmungen der Verletzung von Gerechtigkeitsforderungen muss das Wahlsystem nicht berücksichtigen, da ihnen ein grundlegendes Missverständnis der Funktion eines Verhältniswahlsystems für die Bildung einer regierungsfähigen Koalition zugrunde liegt. Wird aber einem Wahlkreissieger kein Direktmandat zugesprochen, oder wird dem Inhaber eines Listenplatzes, der normalerweise ein Mandat sichern würde, dieses dann nicht zugeteilt, wird eine der grundlegenden Gerechtigkeitsregeln überhaupt gebrochen, nämlich diejenige, Gleiches gleich zu behandeln.

25 Die implizite Kompensation im Rahmen eines kleinen Ausgleichs würde diese Fairnessbedingungen nicht verletzen, da die hier zur Kompensation herangezogenen Mandate ja erst im Rahmen des Ausgleichs entstehen. Es gibt daher keinen ursprünglichen Anspruch auf diese Mandate. 
nicht zu allzu großen Verzerrungen des Länderproporzes kommt. ${ }^{26}$ Dieses Modell verbleibt aber ansonsten vollständig in der Kompensationslogik; die Verteilungsergebnisse sind - mit Ausnahme von 2009 - daher auch identisch zu jenen, die sich unter dem üblichen Kompensationsmodell ergeben würden. Damit bleibt auch hier das Problem bestehen, dass ursprüngliche, kriterienbasierte Mandatsansprüche nicht unangetastet bleiben.

Kompensationsmodelle beruhen auf der Verrechnung zwischen Listen. Damit sie überhaupt funktionieren können, setzen sie daher einen Zwang zur Listenverbindung voraus, da ansonsten ein Anreiz einzelner Landeslisten bestehen könnte, aus der Listenverbindung auszusteigen, um ebendiese Verrechnung zu vermeiden. Ein Zwang zur Listenverbindung könnte aber seinerseits womöglich neue verfassungstheoretische Probleme aufwerfen. Außerdem ließe er sich im Extremfall durch die Gründung eigenständiger Landeslisten umgehen.

Sowohl der Ausgleich als auch die Kompensation der Überhangmandate haben also gewisse Schattenseiten, auch wenn diese im Vergleich zum Problem der Überhangmandate selbst von geringerem Gewicht sind. Der beste Weg wäre daher offensichtlich, die Entstehung der Überhangmandate an sich und damit die gesamte Problematik selbst zu vermeiden. Auf diesem Wege könnte man grundsätzlich die reine Listenwahl oder das Grabenwahlsystem erwägen. Möchte man hingegen den Typus der personalisierten Verhältniswahl aufrecht erhalten, wobei die Personenwahl durch die direkte Wahl von Kandidaten in Wahlkreisen vor sich gehen soll ${ }^{27}$, dann verbleiben eigentlich nur noch die beiden Möglichkeiten, entweder den Anteil der Direktmandate auf deutlich unter 50 Prozent zu senken, etwa auf 35 oder maximal 40 Prozent, oder die Einrichtung von Mehrpersonenwahlkreisen. ${ }^{28}$

Bei Zweipersonenwahlkreisen zum Beispiel würde nicht mehr nur derjenige mit den meisten Stimmen direkt gewählt, sondern die zwei Kandidaten mit den höchsten Stimmenzahlen. Da es auf diese Weise ausgesprochen unwahrscheinlich wird, dass die stärkste Partei noch annähernd alle Direktmandate gewinnt, würde die Anzahl der Überhangmandate entsprechend sinken. Bei Halbierung der Anzahl der Wahlkreise auf circa 150 könnten so - im Gegensatz zu einer Reduzierung der Einerwahlkreise - weiterhin 50 Prozent der Abgeordneten direkt von den Bürgern gewählt werden. Insofern scheint bei Abwägung aller Vor- und Nachteile Einiges für die Einrichtung von Zweipersonenwahlkreise zu sprechen. ${ }^{29}$ Allerdings ist diese Lösung für die anstehende Bundestagswahl nicht mehr realisierbar, da sie einen Neuzuschnitt der Wahlkreise erforderlich machen würde.

Die Einführung eines Einstimmensystems, wie es 1949 für die Bundestagswahl schon einmal galt und in Baden-Württemberg praktiziert wird, würde ebenfalls einige der Überhangmandate vermeiden, nämlich diejenigen, die vornehmlich auf Stimmensplitting zu-

26 Vgl. Richard Peifer / Daniel Lübbert / Kai-Friederike Oelbermann / Friedrich Pukelsheim, Direktmandatsorientierte Proporzanpassung: Eine mit der Personenwahl verbundene Verhältniswahl ohne negative Stimmgewichte, in: DVBL, 127. Jg. (2012), H. 12, S. 725 - 730.

27 Eine Möglichkeit, die Listenwahl mit der Personenwahl zu verknüpfen, bestünde in der Einführung von lose gebundenen Listen. Vgl. Ossip Fürnberg / Danko Knothe, Wahlsiege ohne Stimmenmehrheit: Auswirkungen von verstärktem „Lagersplitting“ auf Mandatsverteilung und Koalitionsoptionen, in: ZParl, 40. Jg. (2009), H. 1, S. $56-74$.

28 Vgl. Joachim Behnke, Überhangmandate und negatives Stimmgewicht: Zweimannwahlkreise und andere Lösungsvorschläge, in: ZParl, 41. Jg. (2010), H. 1, S. 247 - 260.

29 In diesem Simm auch Hubert Kleinert, Anmerkungen zum Wahlrechtsstreit - Ein Problem gelöst, ein anderes bleibt. Oder: Ein Blick über die Grenzen lehrt Gelassenheit, in: ZParl, 43. Jg. (2012), H. 1, S. $185-192$. 
rückzuführen sind. Die Anzahl der Überhangmandate hätte sich bei einem Einstimmensystem 2009 vermutlich auf circa neun bis zwölf reduziert. ${ }^{30}$ Da die Feinheiten des Zweistimmensystems einem großen Teil der Wähler unbekannt sind und es die eben erwähnten Möglichkeiten des strategischen Missbrauchs bietet, kann man einige Vorteile in der Einführung eines Einstimmensystems sehen, das den Typus der personalisierten Verhältniswahl ansonsten in vollem Umfang erhalten würde. ${ }^{31}$

\section{Ein Ausgleichsverfahren auf der Basis der automatischen Methode als mögliche Lösung}

Auch wenn Verfahren, die Überhangmandate gar nicht erst entstehen ließen, offensichtlich die besten Lösungen wären, bleibt für die nächste Bundestagswahl ein Ausgleichsmodell wohl die beste auch noch kurzfristig umsetzbare Lösung. Der Vorbehalt der CDU und FDP gegen das Ausgleichsmodell der SPD allerdings geht dahin, dass dieses das negative Stimmgewicht nicht beseitige, sondern lediglich die Überhangmandate in ihren Auswirkungen ausgleiche, da die Ausgleichsmandate gewissermaßen erst zur Anwendung kommen, wenn das negative Stimmgewicht schon gewirkt hat. In der Tat kommt es beim Ausgleichsmodell der SPD weiterhin dazu, dass mehr Stimmen zum Beispiel weniger Sitze bedeuten könnten. Allerdings wäre damit kein Schaden für den Wähler der entsprechenden Partei verbunden, da dieser Nachteil durch entsprechend weniger Ausgleichsmandate für die Gegenseite neutralisiert würde. Inwiefern dies verfassungswidrig sein könnte, ist zumindest unklar. Es sollte daher nach einer Möglichkeit gesucht werden, den Ausgleich auf eine Weise vorzunehmen, die unstrittig ohne das negative Stimmgewicht auskommt.

Tatsächlich liegt mit dem Entwurf der Linken ${ }^{32}$ ein Modell vor, das in bestimmten Fällen genau diese Funktion erfüllen könnte. Es handelt sich gewissermaßen um eine Kombination aus dem Entwurf der Grünen und dem der SPD. ${ }^{33}$ Überhangmandate der CDU sollten durch Listenmandate kompensiert werden, externe Überhangmandate hingegen durch Ausgleichsmandate. Da bei diesem Modell der Ausgleich der CDU-Überhangmandate im günstigen Fall über ausreichend viele CSU-Überhangmandate gehebelt wird, hätte man die Vorteile eines Ausgleichsmodells, ohne dass auch nur die Spur eines negativen Stimmgewichts auftreten würde. Allerdings müssten hierfür die CDU-Überhangmandate zuerst kompensiert werden; erst danach dürfte die CDU im Rahmen des durch die CSU-Überhangmandate bedingten Ausgleichs wieder zusätzliche Sitze erhalten. Unglücklicherweise aber kann dieser Hebeleffekt nicht garantiert werden. Fallen die CSU-Überhangmandate nicht oder nicht in ausreichender Anzahl an, reduziert sich das Modell auf ein Kompensationsmodell, mit den bekannten Nachteilen. Insofern weist der Entwurf trotz seiner vielversprechenden und interessanten Eigenschaften gewisse Unzulänglichkeiten auf, ganz zu schweigen davon, dass er derjenige ist, der aufgrund seiner Herkunft die geringsten Chancen hat, sich in der politischen Abstimmung durchzusetzen.

30 Vgl. Joachim Behnke / Florian Bader, a.a.O. (Fn. 14).

31 Vgl. Eckhard Jesse, Grundmandatsklausel und Überhangmandate: Zwei wahlrechtliche Eigentümlichkeiten in der Kritik, in: Max Kaase / Hans-Dieter Klingemann (Hrsg.), Wahlen und Wähler: Analysen aus Anlass der Bundestagswahl 1994, Opladen 1998, S. 15 - 41; ders., Reformvorschläge zur Änderung des Wahlrechts, in: APuZ, B 52 / 2003, S. 3 - 11.

32 Vgl. BT-Drs. 17/5896.

33 Vgl. BT-Drs. 17/5895. 
Im Folgenden wird daher ein Ausgleichsmodell vorgestellt, das ohne negatives Stimmgewicht auskommt. Es ist sehr einfach und verständlich, stellt den Parteienproporz vollständig her und gleicht die Verletzung des Länderproporzes zumindest in einem gewissen Umfang wieder aus. Überhangmandate werden vollständig kompensiert, und negatives Stimmgewicht tritt nicht auf. Da es sich um ein Ausgleichsmodell handelt, kommt es auch in keiner Weise zu Verletzungen ursprünglicher, kriterienbasierter Ansprüche an Direkt- oder Listenmandate.

Wandern Sitze bei der Zuteilung von einem Kontingent zu einem anderen, wenn sich die Wählerzahlen ändern, ist es unerheblich, ob es bei dem betreffenden Verteilungsprozess um die Unterverteilung der Sitze auf die Landeslisten wie im alten Gesetz oder die Verteilung von Sitzkontingenten auf die Länder geht. Das negative Stimmgewicht entsteht dann, wenn sich die Sitzzahl einer Partei durch die Abwanderung im Ausgangskontingent nicht verändert - sei es dadurch, dass der Sitzverlust durch Überhangmandate aufgefangen wird, sei es, dass der Sitzverlust zu Lasten einer anderen Partei geht wie im neuen Gesetz -, die Partei aber von einem neu entstandenen Sitz in dem anderen Kontingent profitiert. Das Problem ist daher schon dann gelöst, wenn Sitzverluste aufgrund von Stimmenverlusten in einem Kontingent nicht automatisch mit einem Sitzgewinn in einem anderen Kontingent verknüpft sind. Dies ist bei den bekannten Modellen nur der Fall, weil eine fixe Anzahl von Sitzen verteilt wird. Die einfache Lösung besteht nun in der Verwendung einer automatischen Methode, wie sie zum Beispiel auch in der Weimarer Republik verwendet wurde. ${ }^{34}$ Bei der automatischen Methode wird vorab eine bestimmte Quota, das heißt ein für einen Sitz zu entrichtender „Preis“ in Zweitstimmen, festgelegt. Im Gegensatz zum Wahlrecht der Weimarer Republik wird in dem hier vorgeschlagenen Verfahren die durch die Quota erzielte exakte Sitzzahl auf die nächste ganze Zahl auf die übliche Weise gerundet. Die Sitzzuteilung erfolgt auf die einzelnen Landeslisten direkt. ${ }^{35}$ Da weniger Stimmen für eine Landesliste immer entweder zu gleich vielen oder weniger Proporzmandaten für diese Liste führen, dies aber keinerlei Konsequenzen für die Zuteilung bei irgendeiner anderen Landesliste hat, ist ein negatives Stimmgewicht per definitionem ausgeschlossen. Die automatische Methode hat zudem den großen Vorteil, dass man die Sitzzuteilung wie bisher anhand der Zweitstimmen der zuteilungsberechtigten Parteien berechnen kann und nicht auf Größen wie die Bevölkerungszahl oder die Wahlbeteiligung zurückgreifen muss, um das negative Stimmgewicht zu beseitigen, wie es in einer möglichen Modifikation des neuen Wahlgesetzes der Fall sein könnte. Orientiert man sich zum Beispiel an der Zahl der Wahlberechtigten, führt dies aufgrund der unterschiedlichen Wahlbeteiligung in den Ländern schon zu einer erheblichen Verletzung des föderalen Proporzes noch auf der Ebene der proportionalen Zuteilung der Sitze.

Der einzige Nachteil an der automatischen Methode liegt darin, dass sie zu flexiblen Sitzzahlen führt. Fällt die Wahlbeteiligung geringer aus oder scheitert eine Partei knapp an der Fünfprozenthürde, werden in der Proporzverteilungsphase weniger Sitze verteilt. Dies macht das Verfahren wegen seiner gewissen Unberechenbarkeit bei Politikern in der Regel unbeliebt, man kann diese Eigenschaft aber unter demokratietheoretischen Gesichtspunkten sogar als positiv ansehen, da sie die Parteien belohnt, wenn sie einen guten Wahlkampf geführt haben und eine hohe Wahlbeteiligung erzielen konnten. Da das hier vorgeschlagene Modell aber mit einem Ausgleichsverfahren gekoppelt ist, wird sich die Anzahl der am

34 Vgl. Dieter Nohlen, Wahlrecht und Parteiensystem, Opladen 2009, S. 322 ff.

35 Eine einfache Trennung der Landeslisten würde bei der Verwendung des Sainte-Laguë-Verfahrens das negative Stimmgewicht nicht beseitigen. 
Schluss vergebenen Sitze in der Realität daran orientieren, wie viele Mandate notwendig sind, um die Überhangmandate auszugleichen.

Als pragmatischer Vorschlag für die Quota wurde hier das 0,7fache der Wahlberechtigten, geteilt durch die Sollsitzzahl von 598, genommen. Das heißt aufgrund dieser Quota würden circa 598 Sitze vergeben werden, wenn der Anteil der Zweitstimmen der zuteilungsberechtigten Parteien ungefähr bei 70 Prozent der Wahlberechtigten liegt - die Größenordnung, wie sie bei den letzten Wahlen empirisch zu beobachten war. ${ }^{36}$ Alternative Vorschläge sind aber genauso denkbar, zum Beispiel eine Orientierung an der realen Quota bei der letzten Wahl.

Der Anteil der Zweitstimmen der zuteilungsberechtigten Parteien lag zwischen 1994 und 2005 zwischen 0,73 und 0,76. Auch wenn es in diesem Zeitraum schon eine leicht abnehmende Tendenz gab, so ist der starke Rückgang 2009 auf einen Anteil von nur noch 0,66 auffällig. Dies liegt im Wesentlichen an einer historisch niedrigen Wahlbeteiligung von nur noch circa 70 Prozent. Der Anteilswert von 0,7, der der Berechnung der Quota zugrunde liegt, geht von einer Wahlbeteiligung von circa 73 Prozent aus, bei gleichbleibendem Anteil der Stimmen für nicht vertretene Parteien, damit im Proporzverfahren die regulären 598 Sitze verteilt werden.

Die folgende Modellrechnung bezieht sich auf die Ergebnisse der Bundestagswahl 2009. Im ersten Schritt des Proporzverfahrens wären dabei nach dem vorgeschlagenen Verfahren wegen der relativ niedrigen Wahlbeteiligung nur 560 Mandate zugeteilt worden. Es würden daher erst einmal sogar mehr Überhangmandate entstehen, nämlich 34 statt der tatsächlichen 24. Diese fallen im Wesentlichen in denselben Ländern wie sonst auch in etwas höherer Anzahl an. Die CDU erhielte zum Beispiel in Baden-Württemberg statt zehn nun elf Überhangmandate, die CSU statt drei nun sechs, aber selbst die SPD bekäme nun eines in Bremen. Insgesamt wären für die CDU 27 Überhangmandate, die CSU sechs und die SPD eines angefallen.

Für den Ausgleich wird nun zunächst die Anzahl der benötigten Ausgleichsmandate für die einzelnen Parteien berechnet. Hierfür wird für jede Partei der „Preis“ in Stimmen berechnet, den sie für ihre Mandate „bezahlt“. Tatsächlich bezahlt die CSU, deren 2.830.238 Zweitstimmen insgesamt 45 Mandaten gegenüber stehen, mit 62.894,2 am wenigsten pro Mandat.

\begin{tabular}{|l|r|r|r|r|r|r|}
\hline \multicolumn{1}{|l|}{ Tabelle 1: Verschiedene parteienbezogene Maßzahlen } \\
\hline & \multicolumn{1}{|c|}{ SPD } & CDU & CSU & FDP & Grüne & \multicolumn{1}{c|}{ Linke } \\
\hline Zweitstimmen & 9.990 .488 & 11.828 .277 & 2.830 .238 & 6.316 .080 & 4.643 .272 & 5.155 .933 \\
Proporzmandate & 137 & 161 & 39 & 87 & 65 & 71 \\
Überhangmandate & 1 & 27 & 6 & 0 & 0 & 0 \\
Mandate insgesamt & 138 & 188 & 45 & 87 & 65 & 71 \\
Preis in Zweitstimmen & $72.394,8$ & $62.916,3$ & $62.894,2$ & $72.598,6$ & $71.435,0$ & $72.618,8$ \\
pro Mandat & & \\
\hline
\end{tabular}

Dieser minimale Preis wird nun als neue Quota genommen, das heißt für alle Parteien wird die Anzahl der Mandate errechnet, die ihr aufgrund dieser Quota zustünde. Die Differenz zwischen den so erhaltenen Mandatszahlen und denen, die man nach der ersten Verteilungsrunde erhalten hat, gibt die Anzahl von Ausgleichsmandaten für eine Partei an. 


\begin{tabular}{|l|r|r|r|r|r|c|}
\hline Tabelle 2: Ausgleichsmandate der Parteien \\
\hline & SPD & CDU & CSU & FDP & Grüne & Linke \\
\hline Mandate aufgrund & 159 & 188 & 45 & 100 & 74 & 82 \\
minimalen Preises & 21 & 0 & 0 & 13 & 9 & 11 \\
Ausgleichsmandate & \multicolumn{7}{|l|}{} \\
\hline
\end{tabular}

Zu den 34 Überhangmandaten kommen also noch einmal 54 Ausgleichsmandate hinzu, so dass sich die ursprüngliche Sitzzahl von 560 um 88 auf 648 erhöht. Hätte man für die Anfangsberechnung der Proporzverteilung eine Quota verwendet, die sich auf einen Anteil von 0,66 der Wahlberechtigten bezogen hätte, wären in der Proporzverteilung zuerst 597 Sitze verteilt worden, und es wären insgesamt 669 Sitze in der Endverteilung herausgekommen, also ziemlich genau dieselbe Anzahl wie beim kleinen Ausgleichsverfahren im Rahmen des alten Wahlgesetzes ${ }^{37}$. Die leichte Erhöhung beim hier praktizierten Verfahren ist allerdings nicht zufällig, sondern darauf zurückzuführen, dass der so angewandte Ausgleich „biasfrei“ ist, das heißt es gibt keinen Vorteil des letzten Sitzes.

Das Ausgleichsverfahren löst noch zwei andere Probleme quasi nebenbei. Zum ersten korrigiert es Rundungsfehler, die sich zu einem unangemessen hohen Vorteil oder Nachteil bei der Erstvergabe auf die einzelnen Landeslisten addiert haben können. Zum zweiten behebt es damit auch das Problem der FDP-Zweitstimmen in Bremen. Bei einer Trennung der Landeslisten hat die FDP dort normalerweise keine Chance auf einen Sitz. Da aber beim Ausgleichsverfahren berechnet wird, wie viele zusätzliche Sitze den anderen Parteien bei Anwendung des Minimalpreises der durch die Überhangmandate am meisten bevorzugten Partei zustünden und hierfür ja alle Zweitstimmen der Parteien herangezogen werden, werden so auch die FDP-Stimmen in Bremen einer Verwertung zugeführt.

Hinzu kommt Folgendes: Da die Ausgleichskontingente nicht im Rahmen des üblichen Sitzzuteilungsverfahrens berechnet werden, können sie nun so auf die Landeslisten der Parteien verteilt werden, dass sie den föderalen Proporz stärken. Zuerst wird das größte Kontingent an Ausgleichsmandaten verteilt, das der SPD, dann das der FDP usw. Das erste Ausgleichsmandat geht an das Bundesland, dessen landesbezogener Erfolgswert der schlechteste ist, also das Bundesland, in dem der höchste Preis in Stimmen für einen Sitz bezahlt wird, wenn man alle Sitze und alle Stimmen der Landeslisten aller Parteien in diesem Bundesland betrachtet. In einem iterativen Prozess werden die länderbezogenen Erfolgswerte nach Vergabe jedes Ausgleichsmandats neu berechnet und das nächste Ausgleichsmandat dann an das Land verteilt, das jetzt den höchsten Preis in Stimmen bezahlen muss usw.

Die automatische Methode mit Ausgleich reduziert den Unterschied der Erfolgswerte zwischen den Parteien beziehungsweise den Preis in Stimmen, den diese für ein Mandat zu errichten haben, nahezu auf Null. Zwischen teuerstem und billigstem Preis liegt gerade noch ein Unterschied von etwas mehr als 400 Stimmen. Demgegenüber bleibt der Preisunterschied beim modifizierten Koalitionsentwurf mit über 4.300 Stimmen sehr hoch (vgl. Tabelle 3).

37 Eine Erhöhung des Faktors jedoch auf zum Beispiel 0,73 würde ebenfalls bei 648 landen, da die Endsitzzahl in diesem Fall allein durch die Anzahl der Direktmandate der CSU bestimmt ist und so angepasst wird, dass der Anteil der Direktmandate der CSU ihrem Anteil an Zweitstimmen entspricht. 


\begin{tabular}{|l|c|c|c|c|}
\hline \multicolumn{2}{|l|}{ Tabelle 3: Erfolgswerte für die Parteien } \\
\hline & Automatische Methode mit Ausgleich & \multicolumn{2}{|c|}{ Modifizierter Koalitions-Entwurf } \\
\hline & $\begin{array}{c}\text { Preis in Stimmen } \\
\text { nach Proporz und } \\
\text { Überhang- } \\
\text { mandaten }\end{array}$ & $\begin{array}{c}\text { Endgültiger Preis } \\
\text { in Stimmen }\end{array}$ & $\begin{array}{c}\text { Preis in Stimmen } \\
\text { nach Proporz und } \\
\text { Überhang- } \\
\text { mandaten }\end{array}$ & $\begin{array}{c}\text { Endgültiger Preis } \\
\text { in Stimmen }\end{array}$ \\
\hline SPD & $72.394,8$ & $62.833,3$ & $68.428,0$ & $62.440,6$ \\
CDU & $62.916,4$ & $62.916,4$ & $60.657,8$ & $58.555,8$ \\
CSU & $62.894,2$ & $62.894,2$ & $62.894,2$ & $62.894,2$ \\
FDP & $72.598,6$ & $63.160,8$ & $69.407,5$ & $62.535,4$ \\
Grüne & $71.435,0$ & $62.746,9$ & $67.293,8$ & $62.746,9$ \\
Linke & $72.618,8$ & $62.877,2$ & $66.101,7$ & $62.119,7$ \\
\hline Standardabweichung & $4.851,2$ & 139,0 & $3.380,2$ & $1.651,1$ \\
Größte Differenz & $9.724,6$ & 413,9 & $8.749,6$ & $4.338,4$ \\
\hline \multicolumn{4}{|l|}{ Quelle: Eigene Berechnung. } \\
\hline
\end{tabular}

Auch der föderale Proporz wird mit der automatischen Methode mit Ausgleich deutlich stärker verbessert als mit dem modifizierten Koalitionsentwurf (vgl. Tabelle 4).

\begin{tabular}{|l|c|c|c|c|}
\hline \multicolumn{2}{|l|}{ Tabelle 4: Länderbezogene Erfolgswerte } \\
\hline & \multicolumn{3}{|c|}{ Automatische Methode mit Ausgleich } & \multicolumn{2}{|c|}{ Modifizierter Koalitions-Entwurf } \\
\hline & $\begin{array}{c}\text { Preis in Stimmen } \\
\text { nach Proporz und } \\
\text { Überhang- } \\
\text { mandaten }\end{array}$ & $\begin{array}{c}\text { Endgültiger Preis } \\
\text { in Stimmen }\end{array}$ & $\begin{array}{c}\text { Preis in Stimmen } \\
\text { nach Proporz und } \\
\text { Überhang- } \\
\text { mandaten }\end{array}$ & $\begin{array}{c}\text { Endgültiger Preis } \\
\text { in Stimmen }\end{array}$ \\
\hline Baden-Württemberg & $63.674,0$ & $63.674,0$ & $61.372,6$ & $57.885,5$ \\
Bayern & $68.261,5$ & $63.284,1$ & $65.325,5$ & $61.992,6$ \\
Berlin & $72.180,6$ & $63.519,0$ & $66.165,6$ & $61.075,9$ \\
Brandenburg & $75.614,4$ & $61.211,7$ & $64.272,3$ & $58.429,3$ \\
Bremen & $64.000,6$ & $53.333,8$ & $53.333,8$ & $53.333,8$ \\
Hamburg & $76.754,7$ & $60.307,3$ & $70.358,5$ & $64.946,3$ \\
Hessen & $71.818,5$ & $62.841,2$ & $70.148,3$ & $65.573,4$ \\
Mecklenburg- & $62.743,7$ & $62.743,7$ & $58.262,0$ & $50.979,3$ \\
Vorpommern & $72.654,9$ & $63.848,3$ & $71.423,5$ & $65.843,5$ \\
Niedersachsen & $72.525,5$ & $63.718,8$ & $69.692,4$ & $64.177,2$ \\
Nordrhein-Westfalen & $66.783,9$ & $62.736,4$ & $64.696,9$ & $62.736,4$ \\
Rheinland-Pfalz & $55.661,0$ & $55.661,0$ & $55.661,0$ & $55.661,0$ \\
Saarland & $60.900,9$ & $60.900,9$ & $57.608,9$ & $54.654,6$ \\
Sachsen & $71.287,4$ & $63.366,6$ & $57.029,9$ & $54.314,2$ \\
Sachsen-Anhalt & $64.247,8$ & $61.677,9$ & $64.247,8$ & $64.247,8$ \\
Schleswig-Holstein & $63.873,3$ & $63.873,3$ & $60.511,5$ & $57.486,0$ \\
Thüringen & $5.863,5$ & $3.040,1$ & $5.689,1$ & $4.856,7$ \\
\hline Standardabweichung & $21.093,7$ & $10.539,4$ & $18.089,7$ & $14.864,3$ \\
Größte Differenz & & & & \\
\hline Quelle: Eigene Berechnung. & & & \\
\hline
\end{tabular}




\section{Fazit: Schlechte, weniger schlechte und annehmbare Zwischenlösungen}

Vergleicht man die vorliegenden Entwürfe und die neuen Vorschläge anhand der hier herausgearbeiteten Muss- und Soll-Bedingungen, ergibt sich folgendes Bild:

\section{Tabelle 5: Vergleich der Verfahren in Hinsicht auf die Erfüllung der Muss-und Soll-Bedingungen}

\begin{tabular}{|c|c|c|c|c|c|c|c|c|}
\hline & 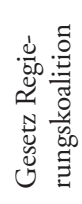 & 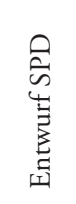 & 莺 & 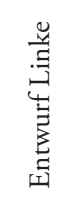 & 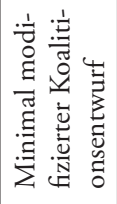 & 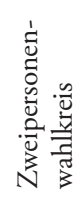 & 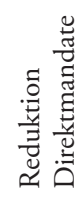 & 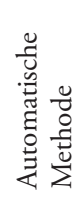 \\
\hline \multicolumn{9}{|l|}{ Muss-Bedingungen } \\
\hline $\begin{array}{l}\text { Kein negatives } \\
\text { Stimmgewicht }\end{array}$ & - & $+1-$ & + & + & + & + & + & + \\
\hline $\begin{array}{l}\text { Maximal } 15 \\
\text { unausgeglichene } \\
\text { Überhangmandate }\end{array}$ & - & + & + & + & + & + & + & + \\
\hline \multicolumn{9}{|l|}{ Soll-Bedingungen } \\
\hline $\begin{array}{l}\text { Vollständige Herstel- } \\
\text { lung des Parteien- } \\
\text { proporzes }\end{array}$ & - & + & + & + & $+/--$ & + & + & + \\
\hline $\begin{array}{l}\text { Keine Verletzung } \\
\text { ursprünglicher } \\
\text { Mandatsansprüche }\end{array}$ & + & + & - & - & + & + & + & + \\
\hline $\begin{array}{l}\text { Geltung des } \\
\text { Länderproporzes }\end{array}$ & $+1-$ & $+/-$ & - & - & $+1-$ & + & + & $++/-$ \\
\hline $\begin{array}{l}\text { Vermeidung unnöti- } \\
\text { ger Aufblähung } \\
\text { des Bundestags }\end{array}$ & $++/-$ & $+/--$ & + & $+/-$ & $+1-$ & + & + & $+1--$ \\
\hline $\begin{array}{l}\text { 50/50 Direkt-/ } \\
\text { Listenmandate }\end{array}$ & $++/-$ & - & + & $++/-$ & $+1-$ & + & - & - \\
\hline $\begin{array}{l}\text { Berechenbare Größe } \\
\text { des Parlaments }\end{array}$ & $+1-$ & - & + & $+/-$ & $+1-$ & $+++/-$ & $+++/-$ & - \\
\hline $\begin{array}{l}\text { Keine strategischen } \\
\text { Anreize }\end{array}$ & - & + & + & + & - & + & + & + \\
\hline
\end{tabular}

Wie man sieht, wäre eine Modifikation des neuen, von der Koalition verabschiedeten Gesetzes, die sich auf das Notwendige beschränken würde, nur hinsichtlich der Muss-Bedingungen besser als das nun beim BVerfG gescheiterte, aber in Hinsicht auf die Soll-Bedingungen keinesfalls geeigneter. Die minimal notwendige Modifikation würde also aus einem schlechten Gesetz nur eines machen, das ein wenig weniger schlecht wäre. Wenig überraschend sind die besten Lösungen diejenigen, die die Überhangmandate erst gar nicht entstehen lassen, also die Reduzierung des Anteils der Direktmandate oder die Einführung von Zweipersonenwahlkreisen, wobei diese Maßnahmen zur Sicherheit immer noch von einer Ausgleichsregelung flankiert werden müssten. Das größte Problem zukünftiger Wahlen werden mit großer Wahrscheinlichkeit die CSU-Überhangmandate werden, da vor allem in 
der mittelfristigen Zukunft damit zu rechnen ist, dass die CSU - ähnlich wie die CDU in Baden-Württemberg - auch Wahlergebnisse von unter 40 Prozent erzielen wird und folglich Überhangmandate in entsprechender Größenordnung anfallen könnten. Mit einem Kompensationsmodell ist diesen nicht beizukommen, der Ausgleich wiederum würde sehr „teuer“ werden. Daher bleibt ein Gesetz, das Überhangmandate möglichst erst gar nicht entstehen lässt, in jedem Fall mittelfristig der einzige sinnvoll zu beschreitende Weg.

Allerdings ist dies wohl nicht mehr zur nächsten Bundestagswahl durchzuführen. Es gibt also derzeit keine realistisch umzusetzende gute Lösung. Insofern bietet sich als der beste gangbare Weg wohl der an, sich jetzt auf ein halbwegs annehmbares Gesetz zu verständigen, dabei aber schon mit zu beschließen, ein besseres alsbald auf den Weg zu bringen. Man könnte natürlich sogar so weit gehen, das endgültige Gesetz schon zu beschließen und lediglich für die nächste Wahl eine davon abweichende Übergangsregelung festzulegen. Als halbwegs annehmbare Lösungen kämen wohl trotz der bestehenden Nachteile ein Kompensations- oder ein Ausgleichsmodell als immer noch beste Alternativen in Frage. Da es erfahrungsgemäß leichter ist, Zugewinne an Sitzen zu verteilen, als vorhandene Sitze umzuverteilen, spricht Einiges für eine Ausgleichslösung. Hier könnte man theoretisch auf den SPD-Entwurf zurückgreifen. Sollte dieser jedoch nicht durchsetzbar sein, stünde mit der automatischen Methode mit angegliedertem Ausgleich eine durchaus brauchbare Alternative zur Verfügung. Ihr „Nachteil“, dass die Anzahl der Sitze nicht fixiert wäre, könnte sich auch als Vorteil dahingehend erweisen, dass dieses Gesetz gar nicht erst als endgültige Lösung konzipiert würde. Das Schlechteste wäre, jetzt ein unzureichendes Gesetz zu verabschieden, das aber womöglich eine gewisse Persistenz aufweisen könnte. Die Versuchung, eine teure und hinsichtlich der Gesamtzahl der Mandate zugleich sichere Ausgleichslösung beizubehalten, wenn sie denn einmal besteht, könnte für die Parteien sehr groß sein. 